\title{
Current status of proton therapy techniques for lung cancer
}

\author{
Youngyih $\operatorname{Han}^{1,2}$ \\ 'Department of Radiation Oncology, Samsung Medical Center, Sungkyunkwan University School of Medicine, Seoul, Korea \\ ${ }^{2}$ Department of Health Sciences and Technology, SAIHST, Sungkyunkwan University, Seoul, Korea
}

Proton beams have been used for cancer treatment for more than 28 years, and several technological advancements have been made to achieve improved clinical outcomes by delivering more accurate and conformal doses to the target cancer cells while minimizing the dose to normal tissues. The state-of-the-art intensity modulated proton therapy is now prevailing as a major treatment technique in proton facilities worldwide, but still faces many challenges in being applied to the lung. Thus, in this article, the current status of proton therapy technique is reviewed and issues regarding the relevant uncertainty in proton therapy in the lung are summarized.

Keywords: Proton therapy, Interplay effect, Intensity modulated proton therapy, Tumor motion, Lung cancer

\section{Introduction}

Lung cancer has been the most frequently diagnosed cancer worldwide and a leading cause of mortality in the United States over the last 50 years [1]. In 2018, lung cancer and breast cancer were reported as the most common cancers worldwide [2] with 2.09 million cases, amounting to $12.3 \%$ of the total number of newly diagnosed cases. Lung cancer was also the most common cause of cancer-related mortality, with 1.76 million deaths reported [3]. In Korea, lung cancer ranked 3rd in cancer incidence and the leading cause of mortality during the last 19 years according to cancer statistics [4]. Although significant advancements in lung cancer treatment have been achieved during the last three decades, it still remains one of the most challenging diseases to cure. The 5-year relative survival rate from 2012 to 2016 was 28.2\%, which is the second lowest among 23 disease sites. The improvement rate of lung cancer patient 5-year survival is much lower than the average rate of all disease sites (16.9\% vs. 29.4\%) [4].

The role of radiotherapy in locally advanced and early stage non-small-cell lung cancer (NSCLC) is well established. Lung cancer is a disease that has the potential to greatly benefit from accurate treatment with proton radiation therapy.

It has been shown that intensity-modulated radiation therapy (IMRT) can improve the overall survival rate compared to three-dimensional conformal external beam radiation therapy (3D-CRT) in stage III NSCLC [5] and achieve a favorable outcome in inoperable NSCLC patients [6]. In Radiation Therapy Oncology Group (RTOC) clinical trials, the survival outcome appears to be equivalent to that of 3D-CRT; however, IMRT was associated with lower rates of severe pneumonitis and cardiac doses. This supports the routine use of IMRT for locally advanced NSCLC [7].

Received 16 December 2019, Revised 24 December 2019, Accepted 26 December 2019.

Correspondence: Youngyih Han, Department of Radiation Oncology, Samsung Medical Center, Sungkyunkwan University School of Medicine, 81 Irwon-ro, Gangnam-gu, Seoul 06351, Korea. Tel: +82-2-3410-2604, Fax: +82-2-3410-2619, E-mail: youngyih@skku.edu (http://orcid.org/0000-0002-1115-5601)

(c) This is an Open Access article distributed under the terms of the Creative Commons Attribution Non-Commercial License (http://creativecommons.org/ licenses/by-nc/4.0/) which permits unrestricted non-commercial use, distribution, and reproduction in any medium, provided the original work is properly cited.

www.e-roj.org 


\section{Particle therapy facilities in clinical operation}

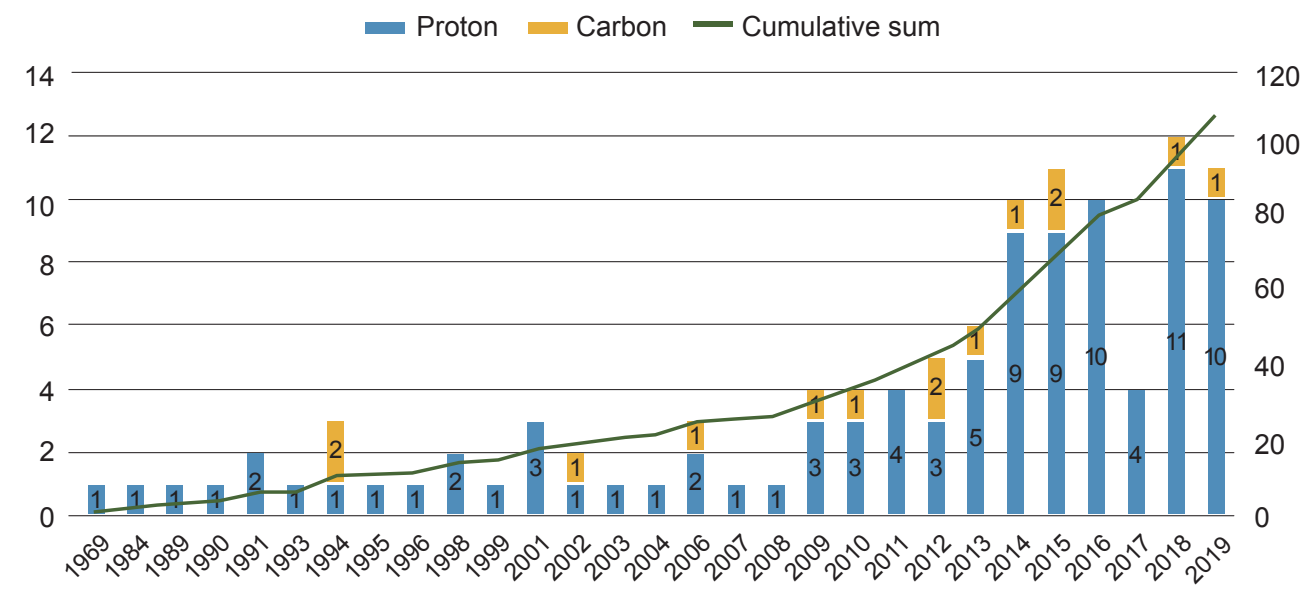

Fig. 1. Number of particle therapy center worldwide. Adapted from Particle Therapy Co-Operative Group [96].

However, the radiation therapy related toxicity is still a major concern in determining radiation therapy strategy. The risk of radiation pneumonitis development is more serious in patients that suffer from chronic pulmonary diseases. Regarding definitive radiotherapy in early-stage NSCLC patients, the radiation pneumonitis ( $\geq$ grade 3 ) is significantly higher and the treatment outcome is worse in patients that suffer from chronic pulmonary diseases such as combined pulmonary fibrosis and emphysema and idiopathic pulmonary fibrosis [8]. In this context, radiation therapy technique, which further limits the radiation dose to the unevolved lung, can potentially contribute to improving the treatment outcome by lowering the treatment-related complications known to substantially contribute to the poor prognosis of patients that suffer chronic pulmonary diseases [9].

Proton therapy can increase the radiation dose to the tumor while simultaneously decreasing the radiation dose to surrounding healthy tissues. For lung cancer treatment, proton therapy could significantly spare the uninvolved lung or decrease the dose to the heart. However, in treating lung cancer with proton therapy, additional considerations associated with the physical characteristic of proton beam need to be made. Considering the uncertainty of proton range in the patient, mitigation of the temporal effects and potential dose discrepancies need to be fully integrated into the proton therapy strategy. The choice in proton beam delivery techniques must also be considered; there are two proton delivery techniques available: passive scattered (PS) proton therapy and active pencil beam scanning (PBS) proton therapy [10].
Phase 2 trials have demonstrated the efficacy of PS proton therapy in unresectable NSCLC treatment by improving the clinical outcome and reducing toxicity compared with historical photon therapy data [11]. The clinical benefit of PS proton therapy in reducing treatment related toxicity in treating locally advanced NSCLC was prospectively studied and compared with that of IMRT. The PS proton therapy was, however, not as effective in reducing $\geq$ grade 3 radiation pneumonitis and local failure as IMRT, but it significantly reduced heart exposure, thereby lowering the cardiac toxicity [12].

In a dosimetry study, PBS proton therapy (IMPT) has been shown to outperform PS proton therapy and IMRT in sparing the lung and nearby critical organs, thereby allowing for further dose escalation for treating stage IIIB NSCLC [13]. Therefore, the IMPT potentially overcomes the limitations of PS proton therapy and outperforms IMRT in reducing treatment related toxicity in the advanced stage of lung cancer and poor prognosis group of patients.

\section{Proton Therapy Overview and Technology}

\section{Overview}

Proton therapy employs the unique feature of proton energy deposition, through which a large percentage of the dose is delivered at the end of the range where particles stop. Therefore, virtually no exit dose is observed. This unique dose deposition feature termed the Bragg peak is especially effective in reducing doses to the regions distal to the Bragg peak; therefore, this characteristic is attractive for treating 


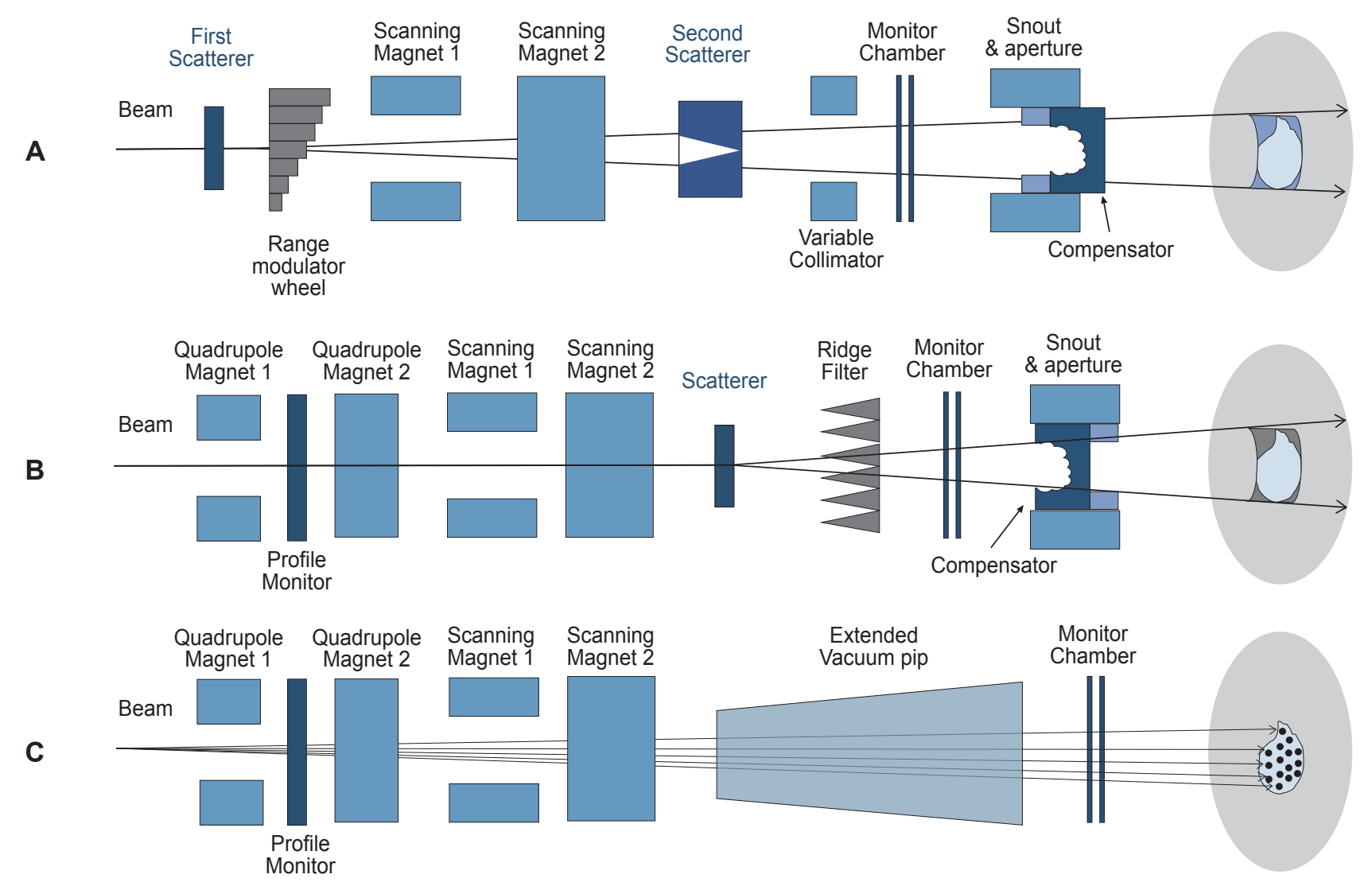

Fig. 2. Schematic diagrams of (A) double scattering nozzle of IBA with block and compensator, (B) Wobbling nozzle of Sumitomo Heavy Industry with block and compensator, and (C) scanning dedicated nozzle of Sumitomo Heavy Industry.

cancer where many critical structures are in close proximity to the target. Planning studies [13] have shown that dose distributions associated with proton therapy allow normal tissue exposure to be lowered in comparison with that of IMRT and potentially open the possibility of dose escalation while maintaining a normal level of tissue exposure.

The first proposal to use high-energy protons for medical treatment was in 1946 by Wilson [14] who was a particle physicist at the Research Laboratory of Physics at Harvard University. The first proton therapy to humans was through pituitary irradiation to control metastatic breast cancer at the Berkeley Radiation Laboratory in 1954 [15] using a proton beam accelerated by a research cyclotron. However, proton therapy eceived approval for clinical application from the US Food and Drug Administration in 1988, and the first hospitalbased proton treatment center opened in California, USA in 1991.

During the last three decades, the number of proton therapy centers has been growing worldwide (Fig. 1), and as of 2018, over 190,000 patients had cumulatively been treated using proton beams.

The techniques used for proton delivery to patients can be categorized into PS proton therapy and PBS proton therapy. Both techniques utilize a mono-energetic proton beam extracted from an accelerator typically with a beam crosssectional diameter of 3-4 $\mathrm{mm}$.

The scattering technique broadens the mono-energetic proton beam laterally, allowing the beam to pass scatterers made with a high atomic number material, which causes the protons to be scattered wide enough to cover the target in a plan orthogonal to the beam path. A double scattering system (IBA, Varian, Hitachi, Mevion) makes the first scattered proton beam to pass the 2 nd scatterer, making a large uniform field.

A wobbling system (IBA, Sumitomo Heavy Industry), which was invented to reduce secondary radiation from scatterers at Berkeley Radiation Laboratory, uses a pair of magnets together with a single scatterer instead of the 2 nd scatterer to make uniform large proton beams (Fig. 2). The accelerated proton beam from the accelerator is rotated along a circular path controlled by magnetic field strengths and then passes 
through a single scatter. Both methods produce fixed sizes of circular irradiation field, therefore a patient block (patient aperture) is necessary to shape the radiation field to fit the maximal tumor cross-sectional area in that orthogonal plan.

Along the depth direction, a range modulator wheel or ridge filter device is used to broaden the narrow width of the Bragg peak to spread out the width enough to cover the tumor. To make the dose conform to the distal of the three-dimensional volume of a tumor, a patient compensator is designed to appropriately compensate for the variation of the distal depth of tumor. However, as shown in Fig. 2, a compensator device is unable to match the dose to proximal of the target volume. This is one of the main disadvantages of PS compared with PBS.

\section{Pencil beam scanning}

The development of PBS technique is considered as a milestone in proton therapy. The motivation towards inventing the PBS technique to replace the scattering system was not only to increase the efficiency by removing the beam-shaping absorbers in the treatment but also to spare normal tissues proximal to the target which is a major deficiency of scattering technique.

The idea to deflect the proton beams using magnets rather than a scatterer for treatment was first suggested by Larsson [16] in 1961 which reported continuous tkrwp scanning (line scanning) using with $187 \mathrm{MeV}$ proton at Uppsala, Sweden. But the clinically applied technique was spot scanning which delivers proton beams spot by spot in a discrete manner which was developed at the National Institute of Radiological Sciences (NIRS, Japan) in 1980 [17]. The first clinical application of PBS for routine treatment was at the Paul Scherrer Institute (PSI) [18]. Currently both of line scanning and spot scanning techniques have been implemented in commercial proton therapy system (spot scanning technique: IBA, Varian, Hitachi, Mevion; line scanning technique: Sumitomo, PSI) for clinical use.

\section{Spot scanning and line scanning}

PBS of spot scanning and line scanning both create broader target coverage from the narrow, mono-energetic proton beam. In the PBS technique, magnets positioned in the upstream of the nozzle deflect each beam and control the beam path to deliver protons across the cross-sectional area of the target. As the beams move through the tumor, proton spots fill the thin thickness (0.7 to 1 sigma; Gaussian shape of spot size) of tumor at the depth where the Bragg peak is made.
Spot scanning fills up each voxel one by one thus delivering the pre-determined dose at each voxel, and pauses the beam while moving across the voxels.

The line scanning does not pause the beam while it paints the dose along voxels in a line. The proton intensity at each voxel is controlled by modulating the proton beam current at the accelerator or beam scanning speed by dynamically controlling magnetic fields in the nozzle.

After completing dose delivery at one slice volume of tumor at that depth, the proton beam energy is changed to deliver the dose to the next slice volume of tumor. The energy changing mechanism is different for different accelerator types (cyclotron vs. synchrotron), and the energy changing time varies from hundreds of milliseconds to a couple of seconds according to the design of energy selection systems and relevant system factors such as power supply of magnets. The energy changing is managed in a step-wise fashion to cover from the distal to the proximal of the target along the beam axis (depth) direction.

Thus, the radiation dose can be painted layer-by-layer. As each layer is filled with an independent and different set of spots, this technique can deliver conformal doses to both the distal and proximal edges of the target, thereby reducing doses to nearby organs at risk (OARs) compared to PS proton therapy. In addition, by varying the intensity of protons to each voxel of the target, the IMPT can be attained.

The aforementioned dose delivery mechanism, in principle, offers a higher level of dose conformity and flexible proton beam delivery compared to the PS approach. Furthermore, it enables intensity-modulated proton therapy [19]. A major obstacle to applying PBS, however, is potential dose deterioration in a moving target when the interplay between the dynamics of beam delivery and organ motion occurs [20].

\section{Uncertainties in Lung Proton Therapy and Overcoming Strategy}

In principle, PBS is superior to scattering but there are large uncertainties in the treatment of lung tumor, which is challenging. The current technologies applicable to PBS treatment of lung tumor are reviewed in this section.

\section{Dose prediction algorithm}

Accurate dose calculations are fundamental in determining treatment strategy. As such, accurate algorithms are inevitably an important factor in reliable treatment as tumor control probability and normal tissue complication probability are 


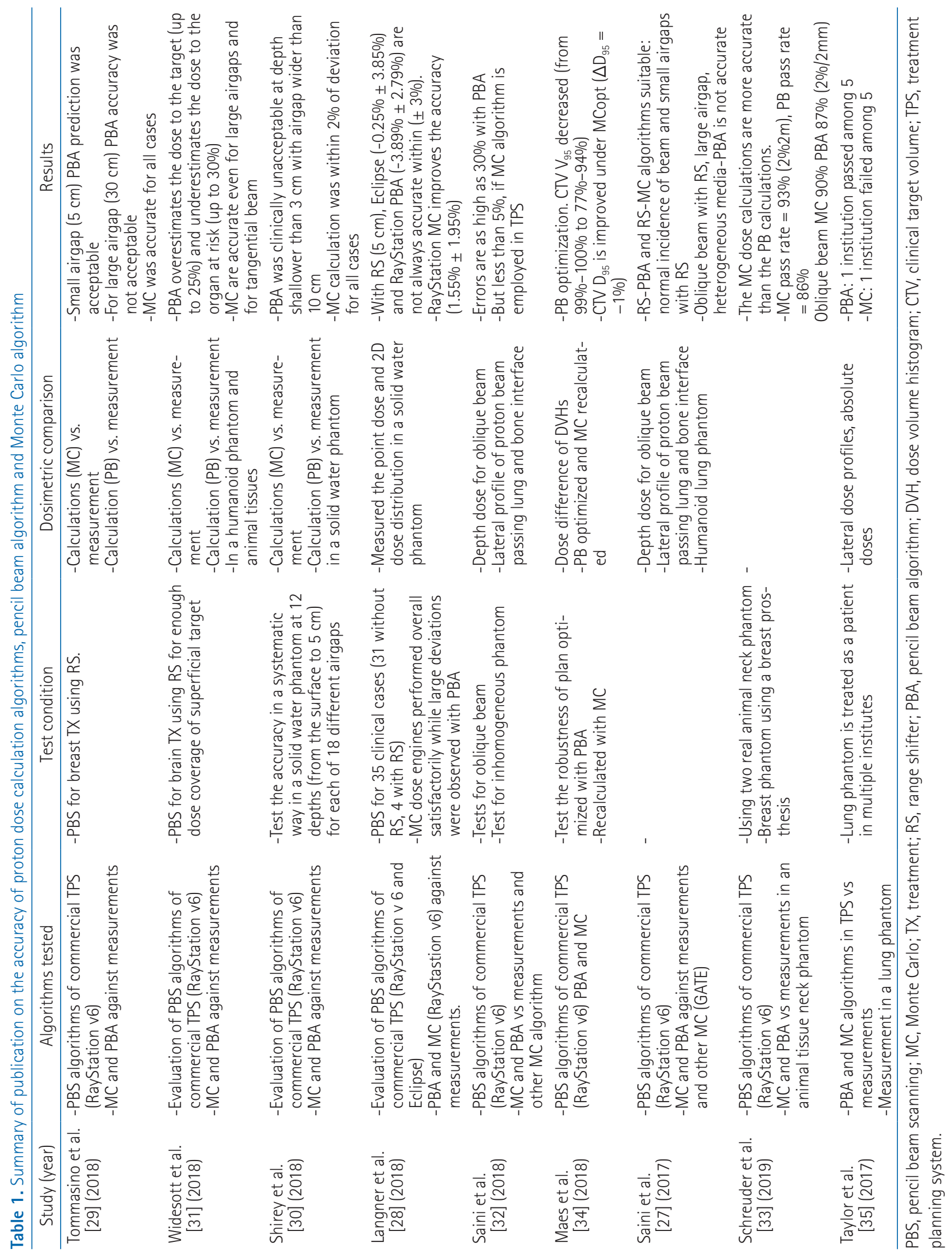


steep functions of absorbed dose in certain disease sites. For clinical use, two different kinds of dose calculation algorithms are available: the analytical algorithm and the Monte Carlo (MC) algorithm (Table 1). Until a couple of years ago, the treatment planning system (TPS) had provided only pencil beam algorithm (PBA), an analytical algorithm, for proton therapy. Therefore, all commercial TPSs provided only the PBA for both PS and PBS treatments. However, as the MC algorithm, which is more accurate in predicting dose distribution in inhomogeneous media, now supports PBS, it is now available in commercial TPSs.

PBAs were developed by Hong et al. [21] in 1996 and subsequently modified and expanded [22-24]. This algorithm models the proton beam as a summation of very narrow pencil beams that are ray-traced individually through the medium and where protons deliver energy by interacting with medium. The delivered dose is therefore computed from the sum of energy delivered by the individual interactions of all pencil beams. On the contrary, MC algorithms track individual particles. For each interaction, energy loss and scattering effects are calculated by randomly sampling the crosssectional data. Until recently, the dose calculation for PBS has been conducted using PBA due to commercial availability and calculation time advantage.

However, as the algorithm does not accurately take into account multiple elastic Coulomb scattering and elastic and inelastic nuclear interactions. PBA fails to provide accurate dose calculation in the case of highly heterogeneous mediasuch as the lung. The PBA models patients as a stack of semiinfinite layers [25]. Thus, the materials encountered by the central axis of each pencil beam are considered to be laterally homogenous slabs along the depth. In real patients with complex anatomies, this approximation limits the accuracy of dose calculation since the lateral inhomogeneities cannot be accurately accounted for in the dose calculation.

On the other hand, MC algorithms method of tracking individual particle trajectories through the material can provide accurate dose calculations in both homogenous and heterogeneous media [26].

\section{Importance of proton dose calculation using MC}

Studies have shown the importance of using MC for certain treatment sites and conditions for PBS treatment.

Recent studies demonstrated that PBA inaccurately calculates dose distribution for proton beams that are oblique to the patient surface [27]. In particular, using the range shifter (RS) with a large air gap, the inaccuracy is clinically unacceptable even in homogenous media. The range shifter shifts the range of proton beams to a shallower depth and is often used to align the dose to a shallow target such as the breast, head and neck and the brain tumor. A study that measured the dose distribution for various air gaps with RS [28-30] proved that an air gap wider than $10 \mathrm{~cm}$ produced a 3\%-11\% dose difference in shallow depths $(<3 \mathrm{~cm})$ for a normal incident beam, while MC algorithm in commercial TPSs predicted a deviation within $2 \%$ even in shallow depths for wide air gaps $[29,30]$.

The lateral dose distribution computed by PBA in the lung and bond interface can be inaccurate by a value as high as $30 \%[31,32]$. The commercial MC TPS enhanced the accuracy of calculating the dose distribution in the lung and bone interface within a $\pm 5 \%$ difference [32] and protons passing through inhomogeneity [33].

The aforementioned deficiency of the PBA algorithm stems from the simplicity of the model which does not fully describe the multiple scattering effects of protons and nuclear halo effects. This results in large-angle scattering particles that appear most prominently for beams with a range shifter and a large air gap to the patient [32]. In the obliquely entering beam, larger spot sizes could result in a bigger deviation between the TPS modeled and the actual doses due to PBA employment of many sub-spots for a single pencil beam.

In animal head tissues, the PBA algorithm overestimated the dose to the target (by up to 25\%) and underestimated the dose to the OAR (by up to 30\%). Therefore, the MC calculation needs to be employed each time the RS is used. In addition, the RS is used with large air gaps and for beams tangential to the patient surface, in which the PBA accuracy is not clinically acceptable [31].

In a comparison of PBA and MC algorithms for PBS of proton therapy improved the accuracy [28-35]. Among the liver, pelvis, brain, head-and-neck, and lung, the difference between PBA and MC calculated doses was largest in the lung, in which the D95 predicted by PBA was 6.5\% lower. Tumor control probability differences can be large for the lungs $(\leq 10.5 \%)$ and the head-and-neck ( $\leq 6.2 \%)$, while smaller for the brain, pelvis, and liver ( $\leq 1.5 \%)$ [36].

\section{Motion incorporated imaging}

The accuracy of a dose calculation is not only determined by the integrity of the implemented algorithm in the TPS, but also the accuracy of the patient data used in the treatment plan. Lung cancer poses a challenging problem in accurate dose calculation in particular due to the highly heterogeneous 

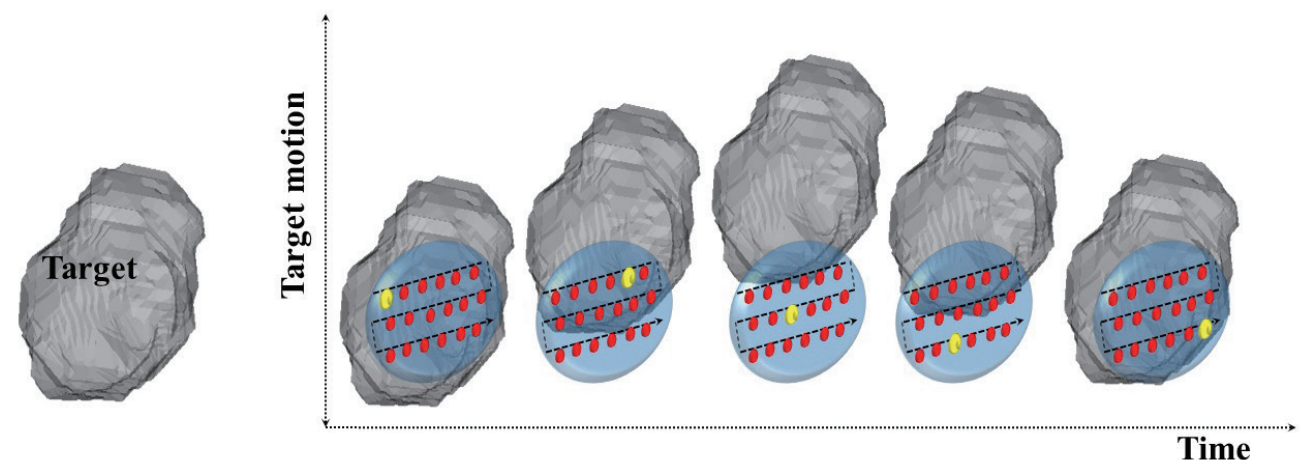

Fig. 3. Schematic diagram showing the beam and target motion interplay. The red colored spots represent the planned spot sequence and the yellow colored spot represent the spot being irradiated at the specific time.

environment of the anatomy. Also computed tomography (CT) data, which represent the spatial and density information on patient anatomy, significantly contribute to the treatment plan in two folds: the geometrical and position information of the target and nearby normal organs presented in the CT contributes to the target volume determination, and the proton's energy deposition is computed using the stopping power at each voxel, which is a variable of the atomic number of the interacting material. For lung cancer, the organ motion increases the uncertainty of both spatial and density information provided by the CT data because respiratoryinduced organ motion compromises CT geometrical accuracy as the CT reconstruction algorithm was developed for static objects.

Four-dimensional computed tomography (4D-CT) is a widely used strategy to reduce respiratory motion artifacts in clinical practice [37]. The 4D-CT technique reconstructs CT images separately into selected allocations according to specified time intervals, or specified amplitudes. Therefore, it allows the representation of volumetric changes of internal organs over respiratory cycle times [38].

Technically, in a cine mode technique (General Electric) projection data at each table position are acquired over the duration of the entire breathing cycle in a cine acquisition mode by rotating the $X$-ray tube multiple times at a fixed table position, and then multiple images sets are reconstructed per table position. The multiple images are synchronized with external respiratory surrogates, which quantify the lung motion. Each of the images is binned into the respiratory cycle phase or amplitude.

However, the 4D-CT images still have artifacts [39], specifically with a high frequency of breathing and large breathing amplitude and consequently influence the size and location of the target tumor in the CT images. For irregular breathing, breathing signals detected by the external surrogates do not accurately represent the internal target motion all the time [40]. This degrades image quality, which may in turn impair quantitative analysis and affect dose calculation accuracy.

In addition, the finer temporal resolution reduces the dosimetric uncertainty in 4D dose calculations for PBS proton therapy [41]. In order to reduce 4D-CT artifacts, several important methods have been reported: (1) using training tools to manage patient breaths in regular pattern [42], (2) using a developed sorting algorithm $[42,43]$ which enhances retrospective sorting techniques such as phase based or amplitude based sorting, and (3) employing internal respiratory signals instead of external surrogates [39]. The basic concept of internal respiratory tracking $[39,43]$, which uses four internal respiratory measures (body area, lung area, air content, and lung density) in the cine images, has been lately integrated into commercial CT systems [39]. For a small tumor, the internal respiratory measured CT produced a smaller volume than that from the CT image obtained using external surrogates. Recently, machine learning using deep neural network approaches are being investigated to reduce the magnitude of 4D-CT artifacts [44].

\section{Interplay Effects}

The dynamic characteristics of the PBS combined with the patient breathing motion can result in the disagreement between the planned dose and the delivered dose, which is termed as an 'interplay effect' (Fig. 3).

The complex radiation therapy beam delivery over the breathing period of patients has been demonstrated to result in a large dose difference due to the interplay of temporal 
characteristics of proton beam positioning and organ positions. These are due to that proton range determined by the relative stopping power ratio, which represents the energy loss of (primary) protons per unit pass length in a medium. Therefore if the radiological depth (or water equivalent depth [WED]) of a proton beam passing through the lung and target tissues changes owing to the movement in/out of dense tissues into the beam path, then protons stop at a depth different from the planed one. This can cause an under dose to the target and over dose to the normal tissues proximal or distal to the beam path.

This interplay effect is observed not only in a PBS treatment, but also PS treatment even though the dose difference was not that pronounced-dose difference of the 95\% of planning target volume (PTV) was less than 3\% [45]. However, in PBS treatment, the deviation can be larger. Early studies [46] on carbon ion therapy for lung tumor, V95\% of the prescribed dose can be received on average $71.0 \%( \pm 14.2 \%)$ for a single treatment fraction. In a recent study on proton spot scanning treatments for pediatric patients, high-dose inhomogeneity $(22.8 \%)$ in one fraction of treatment was detected for Hodgkin lymphoma. Even for 25 fractions in which the dose averaging was expected, the inhomogeneity of dose distribution was 8.7\% [47]. For a single treatment fraction, the interplay effects cause a higher $D_{\max }$ and lower $D_{\min }[48]$ in the target volume, particularly with a small spot size $[48,49]$. Interplay effects narrow the high-dose profile and widen the penumbra across the tumor resulting in a reduction of the 90\% isodose area volume [50].

Dose deviation is less pronounced for larger spot sizes $[20,48,49]$. Considering the fact that spot size differs in proton energy and system design, the significance of dose distribution deterioration depends not only on the energy of proton beam but also on the manufacturers of proton therapy systems. For the same proton energy, the spot size differs for each proton therapy system design such as accelerator type, beam transport system and nozzle design. High energy protons have smaller spot sizes compared to low energy protons. In this regard, the dose degradation can be more severe for carbon beam treatment with a smaller spot size than a proton beam when motion mitigation is not appropriately integrated into the treatment and/or treatment system design [51].

In addition, the scan speed of the proton beam which ranges from $10 \mathrm{~m} / \mathrm{s}$ to $20 \mathrm{~m} / \mathrm{s}$ for high energy beams, or 40 $\mathrm{m} / \mathrm{s}$ for low energy protons in a commercial proton therapy system, can contribute significantly to the dose deterioration by interplay of beam delivery and motion dynamics.
Regarding the tumor motion characteristics, the interplay effect is greater not only when the motion is larger but also when the breathing pattern is irregular [52]. Dose inhomogeneity due to interplay effects is larger for larger asymmetric (longer/shorter exhalation/inhalation) breathing motion [50]. Asymmetric (longer/shorter exhalation/inhalation) breathing motion is one factor that causes dose shifts. When the patient setup is at mid-inhalation, then clinical target volume (CTV) spends more time on the cranial-superior side and causes shifting of delivered dose to the caudalinferior direction. This phenomenon is pronounced for larger asymmetric breathing motion for same motion amplitude of target [50].

However, treatment with conventional fractionations tends to average out the hot and cold spots caused by motion interplay in the lung $[48,49,50,52,53]$, particularly when the initial phase at each treatment is evenly sampled over the entire course of treatment. The interplay effect varies extensively with the initial breathing phase [46-49]; thus, when beam scanning is evenly spaced in time over the breathing cycle, the interplay effects tends to be reduced $[50,53]$. While, for fractionated delivery, local overdose and underdoses to the target may remain for an individual session. These overdose and underdoses will be compensated for in the long run over multiple fractions. Thus, for conventional fractionation, the interplay effects are less pronounced for accumulated dose of entire course of treatment [49].

For a hypofractionation scenario delivering a high dose per fraction, the impact of interplay effect for single large fractional dose was seen less pronounced than that with a regular fractionation dose [52]. This study employed a clinically delivered plan and calculated the interplay by assigning each respiratory phase to each spot according to the recorded time stamp in $4 \mathrm{D}-\mathrm{CT}$. This observation is interpreted that the extension of delivery time for a large dose per fraction increased the probability of spreading the dose evenly throughout all respiratory phases. However, attention must be paid to each individual case. For small tumor sizes and tumors with large motion, a small number of fractions may not provide sufficient repetition to reduce the dose error to a clinically acceptable level. Therefore, the uncertainty in the plan parameters (spot size and temporal characteristics of beam delivery, CTV size, and target motion) needs to be evaluated. Particularly, a motion mitigation strategy needs to be utilized for hypofractionation treatment if necessary [52]. 


\section{Reducing the Motion Induced Uncertainties}

In order to mitigate the motion induced uncertainty, the strategies applicable to three components (plan, patients, and beam delivery) are reviewed and summarized.

\section{Robust plan}

1) Margin recipes for moving the tumor

In order to compliment the deficiency of simple geometric target expansion to form PTV and to account appropriately for proton range uncertainty in the body caused by internal target motion, the beam specific margin concept was introduced. This method explicitly designs a PTV for treatment planning by adding a margin compensating the uncertainty for proximal and distal surface of the target in the beam direction.

Park et al. [54] derived a beam specific PTV (bsPTV), which was originally suggested by Rietzel and Bert [55]. Along each beam direction the variation of water equivalent path length (WEPL) due to tissue misalignment in the presence of setup error or organ motion was calculated for proximal and distal surfaces of the target. After converting the WEPL into radiological thickness based on local tissue density, the beam specific margins are added to the PTV. This method is applicable to single field optimization (SFO) PBS, where each beam is optimized to deliver a uniform dose to the target. The bsPTV strategy has been proven to be robust against the setup error or organ motion assuring 94\% of target dose in test cases. The bsPTV concept was further elaborated to use 4D-CT data to account for details of target motion and shape in each phase of 4D-CT [56].

The beam specific margin concept was extended to be applicable to multi-field optimization IMRT (MFO-IMPT). Graeff et al. [57] developed a 4D water equivalent internal target volume (ITV) that employed the patient 4D-CT data set to analyze target motion and, thus, compute the WEPL of each beam to the target for each phase of breathing motion. The method, which considered the proton fluence of each beam, resulted in a target volume independent of beam directions compatible to IMPT and was implemented into an in-house TPS and tested for use in carbon beam therapy.

\section{2) Robust optimization}

For MFO-IMPT, each beam is modulated in such a manner that allows each beam to limit the dose to critical organs, but also allows a uniform dose distribution to be achieved in the target when all beams are combined together. In this way, each individual beam, actually produces a highly inhomogeneous dose distribution in the target. Thus, MFO-IMPT is more sensitive to the target motion and any uncertainty involved. To make the IMPT plan robust, robust optimization algorithms have been proposed.

The basic idea of the robust optimization is that the optimizer takes into account expected uncertainty in the planning stage by considering the worst case scenario or all possible scenarios, which can be caused by uncertainties such as patient setup, beam range, target and internal organ motion.

Three-dimensional robust optimization (3D-R0) algorithms incorporate setup errors and/or range certainties directly into the optimization of an intensity modulated beam. The probabilistic approaches [58] determine error probability distribution and compute possible dose distributions by randomly sampling setup and range uncertainty scenarios and optimizes proton fluence. Therefore, these approaches require a large amount of computational resources [58].

The optimization algorithm, which considers only the worst-case scenario, was able to avoid intensive computation. The minimax optimization [59] considers only physically realizable scenarios, i.e., evaluating a number of scenarios and considering the worst one. The Voxel-wise worst-case robust optimization method, additionally computes 8 cases of errors ( 6 position shifts and 2 cases of range shifts) together with the normal dose distribution not taking into account the uncertainties. The proton beamlet are optimized so that the lowest dose voxel in the CTV and the highest dose voxel outside the CTV are mitigated [60].

The minimax algorithm improves the robustness of the (SFO) scanning plan in lung, paraspinal, and prostate cancer cases [59]. The worst-case optimization reduces uncertainty in the para-spinal IMPT plan [60]. For distal edge tracking IMPT plans, 3D-RO improves plan robustness; however, the extent of improvement varies according to the disease sites [61]. Fredriksson and Bokrantz [62] evaluated the three worstcase methods, voxel wise optimization [58], object wise optimization [63], and minimax optimization [59] with respect to treatment planning for prostate cancer. With systematic setup uncertainties, all methods produced robust plans only when the conflict between target coverage and normal tissue sparing was small; however, in other cases, each method exhibited different shortcomings. Therefore, no particular method was considered to be superior to the others under all circumstances [62].

In order to take into account for the uncertainty of target 
motion, 4D-RO was suggested. The 4D-RO explicitly included the organ motion and shape change by using a $4 \mathrm{D}-\mathrm{CT}$ in the plan instead of a 3D-CT. The 4D-CT provides critical organ motion as well as tumor motion so that target and OAR shape and position change can be incorporated into the optimization.

Graeff's algorithm [57,64], which includes the organ motion induced by breathing, was extended further to include range uncertainty due to $\mathrm{CT}$ number related uncertainties such as $\mathrm{CT}$ number statistical fluctuations, the conversion of CT numbers to proton stopping power ratios, and the artifacts in the CT image, and setup uncertainty at each of the ten phases of the $4 \mathrm{D}-\mathrm{CT}$ dataset $[65,66]$. Liu et al. [66] deformed all 10 phases of the doses to a reference phase $C T$, and then the setup and range uncertainty were evaluated for the cumulative $4 D$ dose distribution. Ge et al. [65] considered the setup and range uncertainty at each breathing phase and simultaneously optimized the dose distribution. The 4D-RO plan is shown to be more robust compared to the $3 \mathrm{D}-\mathrm{R} 0$ plans for 10 lung cases in which various motion ranges were used (2.5-12.4 mm) [65], which resulted in better target coverage, dose homogeneity, and plan robustness in the face of uncertainties. The 4D-RO plans resulted in interplay-resistant plans for target coverage while producing comparable dose distributions for normal tissues in lung cases [66].

The aforementioned 4D-RO considered organ motion and shape change but did not explicitly incorporate the time structure of the delivered beam dynamics in the beam optimization as the entire beam dose was computed and accumulated over the 4D-CT images. In order to incorporate the time structure into the optimization, Graeff et al. [67] subdivided the target into sectors which was matched to each phase of the 4D-CT. A group of spots was assigned to each sector of the target which corresponds to each phase of breathing motion. All groups of spots are then optimized simultaneously to determine the particle numbers at each spot and thus resulted in a homogeneous dose for the target. The beam spots need to be delivered to the correct phases; therefore, during the beam delivery, the spot sequence needs to be sorted at the treatment control system and must be synchronized to the breathing motion [64]. This delivery strategy was facilitated by the on-line phase-controlled rescanning developed for carbon ion therapy [68].

Engwall et al. [69] suggested a 4D-RO method, which included the time structures of the beam delivery and organ motion in the $4 \mathrm{D}$ robust optimization process to generate plans that are robust against interplay effects while it does not require additional implementation in the treatment control system. The algorithm acquired an accurate delivery time structure for ordered spot energies, positions, and weights from the proton therapy system (PTS) for every 10 iterations in the plan optimization and incorporated the time structure into the optimization. It also updated the spot weights. This was possible by connecting to the PTS simulation system that provides the spot delivery time characteristics. As for the optimization methods, partial beam doses of each phase of $4 D$ CT are deformed to the reference phase CT and accumulated on a reference phase, while a minimax optimization is performed to take all scenarios into account simultaneously. The effectiveness of the method was assessed for IMPT for lung cancer and the efficiency was proven particularly for larger tumor motion.

\section{Patient's motion management}

One approach to reduce the internal organ motion is to ask a patient to hold their breath or compress the patient's abdomen to reduce the breathing amplitude.

For lung cancer patients, studies tested the abdominal compression using 4D-CT and 4D-CT/ positron emission tomography (PET) imaging.

\section{1) Abdominal compression}

The abdominal compression could be useful for stereotactic body radiation therapy (SBRT) of lung or liver treatments and has been used in X-rays SBRT.

For lung cancer patients, the abdominal compression efficacy was assessed using 4D-CT, PET, and cone beam computed tomography (CBCT) imaging. Using PET imaging, the efficacy of abdominal compression (BodyFIX Diaphragm Control; Elekta, Stockholm, Sweden) in reducing the lung motion was verified. Reducing the tumor motion lowered the mismatch between $\mathrm{CT}$ and the corresponding attenuation corrected PET images, and it increased the standardized uptake value (SUVmax) and SUVmean for all tested lung cases [70].

Using 4D-CT, abdominal compression device (ACD) efficacy on respiratory-induced motion was assessed [71]. The gross tumor volume (GTV), heart, and lung motions were not significantly affected by $A C D$, but the efficacy was patient specific. GTV motion was reduced in 3 cases while motion was increased in 5 cases, and no effects were observed in the remaining 9 patients. This was potentially due to the patient specific physical parameters and/or the difference in tumor location. The abdominal compression was effective in reducing motion of the tumor close to the diaphragm. However, it was less effective and even increased the motion of tumor in the 
middle and upper locations in the lung $[72,73]$.

As for the daily setup reproducibility, analyzing the CBCT image acquired with the ACD for the lung SBRT, the GTV volume and position were quantified. The daily CBCT included tumor motion amplitude due to the 1 minute of image acquiring time. The daily variation in tumor position was larger than that in the cases where the compression device was not used; a greather than or equal to $5 \mathrm{~mm}$ variation was seen in more fractions with the ACD than in those without (17\% vs. $9.2 \%)$. The largest variance in the variation was in the cranialcaudal direction [73].

Abdominal compression was effective in suppressing the motion of the tumor in the lower lob, but was not sufficient to replace $4 \mathrm{D}-\mathrm{CT}$ with $3 \mathrm{D}-\mathrm{CT}$. With $\mathrm{ACD}$, both $3 \mathrm{D}-\mathrm{CT}$ simulations and daily CBCT significantly underestimated the full range of tumor motion [74]. Special care must be taken while designing and positioning the device as any interference caused by the high density part of the device in the beam path can shift the range of the proton beam.

\section{2) Breath hold}

Breath hold $(\mathrm{BH})$ or deep inspiration breath hold $(\mathrm{DIBH})$ can be achieved by patients voluntarily or with computer-controlled devices which assists in hold the breath by blocking airways and/or feedback breathing signals. DIBH was considered to be beneficial by not only immobilizing lung tumor but also reducing the lung density, thereby allowing the normal lung volume to be reduced in the high dose region [75].

With a patient using DIBH voluntarily with the aid of a visual guide, the systematic and random errors, and interbreath-hold position uncertainty in the lymph node and target positions were assessed using fluoroscopic images [76] or three consecutive DIBH CTs [77]. Voluntary DIBH substantially reduced the motion, but it did not completely eliminate the motion with finite values of mean inter-breathhold shift of -0.2 to $-0.3 \mathrm{~mm}$ [76] and $4.8 \pm 2.8 \mathrm{~mm}$ [77] for repeated breath holds. The largest systematic deviation of all the mean marker positions was in the craniocaudal direction $(0.8 \mathrm{~mm})$ and random errors (root-mean-square of the intra$\mathrm{BH}$ standard deviation) during the $\mathrm{DIBH}$ were less than 0.5 $\mathrm{mm}$ [76] or $1.7 \pm 1.4 \mathrm{~mm}$ [78]. In a large prospective cohort study of locally advanced NSCLC patients treated using DIBH, the overall DIBH compliance was high (72\%). The systematic and random errors of peripheral tumor and lymph nodes were small in consecutive DIBHs; however, the residual motion must be included in the PTV margins [78].

Patient compliance is important for the reproducibility of DIBH treatment. A moderate and comfortable level of breath holding with the aid of visual feedback increases reproducibility and thus, the consistency in the breath-hold lung tumor position and volume can be achieved [79].

3) Robustness of breath hold plans

The feasibility of treating locally advanced NSCLC with IMPT in visually guided voluntary DIBH was tested by acquiring additional CT images (3-9 times) during the course of proton therapy. The 4D plans considering both patient intra- and inter-fractional motions were robust against the inter- and intra-variation of tumor motion since the dose difference in the target and OAR was minimal for most patients. Treatment was deliverable in less than or equal to 3 breath holds in 9 patients. For larger tumors $\left(>200 \mathrm{~cm}^{3}\right)$, treatment with $\mathrm{BH}$ was unfeasible due to long beam delivery times [80]. However the beam delivery time varies according to PBS system design and accelerator type; therefore, the feasibility needs to be determined at each individual PTS system.

The DIBH assisted by computer-controlled BH been used widely used for breast cancer radiation therapy. An active breathing coordinator $(A B C)$ is a computer-controlled device that temporarily immobilizes the patient's breathing [81] by closing the airway. Thus, it freezes the tumor and organ motion.

The stability of DIBH assisted by $A B C$ was assessed by quantifying intra- and inter-fractional variations of organ for NSCLC patients. Intra-fractional variation of tumor motion was assessed using fluoroscopy videos [82], repeated breath hold CTs $[83,84]$ for NSCLC patients undergoing radiation therapy, and magnetic resonance imaging (MRI) for healthy volunteers $[85,86]$. The intra-fractional mean variation ranged from 0.6 (range, 0 to 3 ) to 0.3 (0 to 1 ) $\mathrm{mm}, 0.6$ (0 to 2) to 1.7 (0.1 to 6.0 ) $\mathrm{mm}$, and 1.7 (0.2 to 5.0 ) to 1.5 (0 to 5.2 ) $\mathrm{mm}$ [83] in the right-left (RL) superior-interior (SI), and anterior-posterior (AP) directions, respectively [82].

The inter-fractional variations were measured using repeated CT scans [82,83], daily CBCT [84] for lung cancer patients, and repeated MRI imaging [85] for healthy volunteers. The inter-fractional variations were larger than intra-fractional variations [79,84-86]. $A B C$ devices assisted $B H$ was more stable than self-controlled $\mathrm{BH}$. The average lung volume variation with $A B C$ was $1.8 \%$ of the measured lung volumes $(99 \mathrm{~mL})$ and was smaller than the $4.1 \%(226 \mathrm{~mL})$ variability obtained with the self-sustained $\mathrm{BH}$ [85].

To deliver a plan robust to intra- and inter-fractional variation of $A B C$ controlled $B H s$, a robust optimized IMPT plan 
mitigating the uncertainty under $A B C$ controlled delivery is considered a safe approach for PBS lung treatment [86].

\section{4D beam delivery}

Irradiating radiation to a static target in conjunction with $\mathrm{BH}$ is one of many possible strategies. For patients who are not eligible for $\mathrm{BH}$, other beam delivery techniques are necessary to mitigate target motion in the case of a free breathing patient. Possible technologies are beam gating, rescanning, and tumor tracking through real-time imaging techniques.

\section{1) Beam gating}

Beam gating method consists of delivering a dose to a target only when localized in a predetermined position within a gating window. Therefore real-time monitoring of the target position is essential. The patient's breathing must be monitored, either through use of an external surrogate or through internal target monitoring via real-time $\mathrm{X}$-ray imaging (fluoroscopy imaging) [87]. To achieve the clinically acceptable level of dose preservation within an acceptable treatment time, it is essential to determine the most reproducible and stable phase and suitable gating window [88].

In addition to the patient related factors, beam delivery characteristics, in particular, parameter choices, affects total delivery time and as an extension quality of treatment. Total irradiating time depends on the specific characteristics of the PTS such as beam current, spill intensity in the case of synchrotron use, energy chaining time, and planning parameters especially total number of spots. Assigning finer spot spacing during IMPT optimization generates a higher number of spots and longer treatment time.

Other factors to be considered are accelerator type and energy selecting design structure which determine the on/ off characteristics of the beam. A proton beam accelerated in a cyclotron is relatively continuous despite energy changes from one to the next taking a couple of hundreds milliseconds for recent PTS and 1-2 seconds for older systems. The proton beam from a synchrotron is a pulsed beam which is extracted periodically in the flat-top phase of accelerator. For a fixed flat-top system, a finite period ( 1 second) of flat-top is followed by a longer period (4-5 seconds) of spill pause [89]. Thus, the synchronization of the beam spill and flat-top phase with the gate-on signal is crucial in executing gated treatment with synchrotron PTS. Thus, innovative technologies such as variable flat-top phase operation [90], and multiple gated irradiation function [87] have been developed to enhance synchronization in synchrotron PTS.
In a gated treatment, finite residual motion within the gating window may still trigger interplay effects. Therefore, when assigning the gating window in consideration of treatment time, it is important to consider additional motion mitigating strategies.

\section{2) Rescanning}

Rescanning (or repainting) delivers one fraction of the dose in multiple repeat scanning. Fluctuating minor over and underdoses to the target ultimately achieve a statistical averaging out effect at the target dose. The volumetric rescanning and layer-by-layer rescanning (or layered rescanning) are distinguished in scanning methodologies $[91,92]$. Volumetric rescanning delivers a subfractional daily dose ( $d / n$, where $n$ is rescanning number, $d$ is daily dose) to the whole volume of target on each scan and repeats the 3D depth scanning multiple times $[91,93]$. The layered rescanning method delivers a subfractional dose at each energy layer and repeats this multiple times at that isoenergy layer and then moves to the next layer [92]. Rescanning is only clinically feasible with a highly dynamic and precise system that support fast energy changing, ultrafast beam scanning and precise beam positioning.

The efficacy of rescanning depends on the time characteristics of the PBS delivery system. The layered rescanning is superior to volumetric rescanning, particularly for slow scanning systems, in achieving a homogenous dose distribution in a clinically acceptable beam delivery time (300 seconds). However, layered rescanning appears to be more sensitive to the starting phase [92]. The simulation of layered rescanning for moderate motion $(5 \mathrm{~mm})$ shows that continuous line scanning outperforms spot scanning due to its high rescanning rate within a short treatment time [91]. By eliminating the dead time between spots, continuous line scanning achieves better speed. It's dose distributions are comparable with those of discrete spot scanning, with only a modest degradation of lateral penumbra in the scanning direction-which is one of the drawbacks of the rescanning method with free breathing. For large tumor motion $(>1 \mathrm{~cm})$ rescanning needs to be combined with gating or $\mathrm{BH}$ to achieve the desired level of dosimetric benefit [93].

\section{3) Tumor tracking}

Aforementioned motion mitigating technologies are in clinical use, but tumor tracking still remains a research topic in proton therapy. Real-time tumor tracking for proton therapy could be the most advanced strategy for tumor motion mitigation, 
but it involves many challenges. To track the target position, accurate real-time 3D detection of the tumor position, radiological path length with high spatial and temporal resolution is essential [94]. Fast spot sequence or spot scanning speed adjustment capability in the lateral dimension and/or fast range adaptation of the particles to shift the Bragg peak position in depth are requisite technologies for tumor tracking. The time delay associated with acquiring and processing images, allowing communication between devices and adjusting beam position according to the target position is unavoidable. Yet, latency must be managed to be shorter than temporal resolution of motion detection. Therefore, in addition to highly accurate motion detecting ability, quick-responding PTS systems must be developed in order to realize real-time tumor tracking for proton therapy.

\section{Conclusion}

Proton therapy is in a stage of rapid growth. As of 2018 more than 190,000 patients have been treated with proton beam [95]. By 2030 the number of patients treated with proton therapy is expected to grow to be over 300,000 . However, the clinical evidence clearly supporting broad use of proton therapy is not yet clear in many tumor sites [97]. Particularly in the lung, the clinical benefit in reducing lung toxicity and improving treatment outcomes with PS is not evident compared with IMRT. However, it is clear that PBS proton therapy does have a dosimetric advantage over IMRT.

Yet, the current PBS techniques of mitigating the motion related uncertainty are not fully mature. As for the machine performance, the scanning speed and energy layer switching time need to be further improved for efficient delivery of rescanning and gating treatments and for the realization of the real-time tumor tracking treatments. In addition, the artifacts in 4D-CT relevant to irregular and abnormal breathing of lung patients need to be overcome. Further research on $4 \mathrm{D}$ in room imaging such as $4 \mathrm{D}-\mathrm{CBCT}$ will contribute to the accurate localization of moving targets.

Since MC algorithm is commercially available, implementing $\mathrm{MC}$ algorithm in each clinic is desirable for minimizing the dosimetric uncertainty relevant to the complicate anatomy in lung cancer treatment. Patient's motion management is challenging. Due to diverse characteristics associated with the diseases states of lung cancer patients, any single strategy is not able to meet to all patients. Therefore, multiple strategies reviewed in this article shall be implemented in each clinic to meet each patient' specific needs.
In order for the promise of PBS as treatment technique to be fully realized, further development on motion mitigation strategies, dose calculation algorithms fully considering motion and making plan robust, and more efficient PTS systems are required. The advancement in such technologies are anticipated to ultimately translate the dosimetric advantages to clinical benefit.

\section{Conflict of Interest}

No potential conflict of interest relevant to this article was reported

\section{Acknowledgments}

This work was supported by the National Research Foundation of Korea funded by the Ministry of Science, ICT \& Future Planning (No. 2019M2A2B4096537). Author is grateful to Dr. Jae Myung Noh and Dr. Heesoon Sheen for carefully reading this manuscript and giving suggestions. Special thanks go to Mr. Jinhyeop Lee and Mr. Sang Woon Jeong for making figure images presented in this article.

\section{References}

1. American Cancer Society. About lung cancer [Internet]. Atlanta, GA: American Cancer Society; c2019 [cited 2019 Dec 26]. Available from: https://www.cancer.org/content/dam/CRC/ PDF/Public/8703.00.pdf.

2. World Cancer Research Fund International. Worldwide cancer data [Internet]. London, UK: World Cancer Research Fund International; 2018 [cited 2019 Dec 26]. Available from: https://www.wcrf.org/dietandcancer/cancer-trends/ worldwide-cancer-data.

3. World Health Organization. Cancer [Internet]. Geneva, Switzerland: World Health Organization; 2018 [cited 2019 Dec 26]. Available from: https://www.who.int/news-room/factsheets/detail/cancer.

4. Jung KW, Won YJ, Kong HJ, Lee ES. Cancer statistics in Korea: incidence, mortality, survival, and prevalence in 2016. Cancer Res Treat 2019;51:417-30.

5. Harris JP, Murphy JD, Hanlon AL, Le OT, Loo BW Jr, Diehn M. A population-based comparative effectiveness study of radiation therapy techniques in stage III non-small cell lung cancer. Int J Radiat Oncol Biol Phys 2014;88:872-84.

6. Sura S, Gupta V, Yorke E, Jackson A, Amols H, Rosenzweig KE. Intensity-modulated radiation therapy (IMRT) for 
inoperable non-small cell lung cancer: the Memorial SloanKettering Cancer Center (MSKCC) experience. Radiother Oncol 2008;87:17-23.

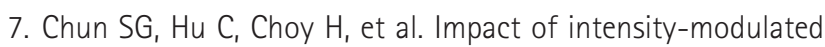
radiation therapy technique for locally advanced non-smallcell lung cancer: a secondary analysis of the NRG oncology RTOG 0617 randomized clinical trial. J Clin Oncol 2017;35:5662.

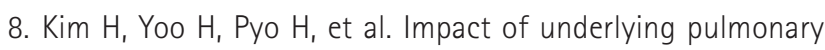
diseases on treatment outcomes in early-stage non-small cell lung cancer treated with definitive radiotherapy. Int J Chron Obstruct Pulmon Dis 2019;14:2273-81.

9. Watanabe $A$, Higami $T$, Ohori $S$, Koyanagi $T$, Nakashima $S$, Mawatari T. Is lung cancer resection indicated in patients with idiopathic pulmonary fibrosis? J Thorac Cardiovasc Surg 2008;136:1357-63.

10. St James S, Grassberger C, Lu HM. Considerations when treating lung cancer with passive scatter or active scanning proton therapy. Transl Lung Cancer Res 2018;7:210-5.

11. Chang JY, Verma $V$, Li M, et al. Proton beam radiotherapy and concurrent chemotherapy for unresectable stage III non-small cell lung cancer: final results of a phase 2 study. JAMA Oncol 2017;3:e172032.

12. Liao Z, Lee JJ, Komaki R, et al. Bayesian adaptive randomization trial of passive scattering proton therapy and intensitymodulated photon radiotherapy for locally advanced nonsmall-cell lung cancer. J Clin Oncol 2018;36:1813-22.

13. Zhang $X$, Li $Y$, Pan $X$, et al. Intensity-modulated proton therapy reduces the dose to normal tissue compared with intensitymodulated radiation therapy or passive scattering proton therapy and enables individualized radical radiotherapy for extensive stage IIIB non-small-cell lung cancer: a virtual clinical study. Int J Radiat Oncol Biol Phys 2010;77:357-66.

14. Wilson RR. Radiological use of fast protons. Radiology 1946:47:487-91.

15. Lawrence $J H$, Tobias CA, Born JL, et al. Pituitary irradiation with high-energy proton beams: a preliminary report. Cancer Res 1958;18:121-34.

16. Larsson B. Pre-therapeutic physical experiments with high energy protons. Br J Radiol 1961;34:143-51.

17. Kanai T, Kawachi $K$, Kumamoto $Y$, et al. Spot scanning system for proton radiotherapy. Med Phys 1980;7:365-9.

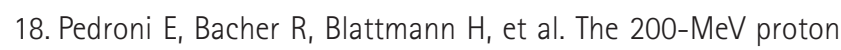
therapy project at the Paul Scherrer Institute: conceptual design and practical realization. Med Phys 1995;22:37-53.

19. Lomax A. Intensity modulation methods for proton radiotherapy. Phys Med Biol 1999;44:185-205.
20. Phillips $M H_{1}$ Pedroni $E_{1}$ Blattmann $H_{1}$ Boehringer $T$, Coray $A_{t}$ Scheib S. Effects of respiratory motion on dose uniformity with a charged particle scanning method. Phys Med Biol 1992;37:223-34.

21. Hong $L$, Goitein $M$, Bucciolini $M$, et al. A pencil beam algorithm for proton dose calculations. Phys Med Biol 1996;41:1305-30.

22. Schaffner B, Pedroni E, Lomax A. Dose calculation models for proton treatment planning using a dynamic beam delivery system: an attempt to include density heterogeneity effects in the analytical dose calculation. Phys Med Biol 1999;44:27-41.

23. Russell KR, Isacsson $U$, Saxner $M$, et al. Implementation of pencil kernel and depth penetration algorithms for treatment planning of proton beams. Phys Med Biol 2000;45:9-27.

24. Soukup $M$, Fippel $M$, Alber M. A pencil beam algorithm for intensity modulated proton therapy derived from Monte Carlo simulations. Phys Med Biol 2005;50:5089-104.

25. Szymanowski H, Oelfke U. Two-dimensional pencil beam scaling: an improved proton dose algorithm for heterogeneous media. Phys Med Biol 2002;47:3313-30.

26. Paganetti $\mathrm{H}$. Monte Carlo simulations will change the way we treat patients with proton beams today. $\mathrm{Br} J$ Radiol 2014;87:20140293.

27. Saini J, Maes D, Egan $A$, et al. Dosimetric evaluation of a commercial proton spot scanning Monte-Carlo dose algorithm: comparisons against measurements and simulations. Phys Med Biol 2017;62:7659-81.

28. Langner UW, Mundis M, Strauss D, Zhu M, Mossahebi S. A comparison of two pencil beam scanning treatment planning systems for proton therapy. J Appl Clin Med Phys 2018;19:156-63.

29. Tommasino F, Fellin F, Lorentini $S$, Farace P. Impact of dose engine algorithm in pencil beam scanning proton therapy for breast cancer. Phys Med 2018;50:7-12.

30. Shirey RJ, Wu HT. Quantifying the effect of air gap, depth, and range shifter thickness on TPS dosimetric accuracy in superficial PBS proton therapy. J Appl Clin Med Phys 2018;19:164-73.

31. Widesott $L$, Lorentini S, Fracchiolla F, Farace $P$, Schwarz M. Improvements in pencil beam scanning proton therapy dose calculation accuracy in brain tumor cases with a commercial Monte Carlo algorithm. Phys Med Biol 2018;63:145016.

32. Saini J, Traneus E, Maes D, et al. Advanced proton beam dosimetry part I: review and performance evaluation of dose calculation algorithms. Transl Lung Cancer Res 2018;7:171-9.

33. Schreuder AN, Bridges DS, Rigsby $L$, et al. Validation of the RayStation Monte Carlo dose calculation algorithm using a realistic lung phantom. J Appl Clin Med Phys 2019;20:127-37 
34. Maes D, Saini J, Zeng J, Rengan R, Wong T, Bowen SR. Advanced proton beam dosimetry part II: Monte Carlo vs. pencil beam-based planning for lung cancer. Transl Lung Cancer Res 2018;7:114-21.

35. Taylor PA, Kry SF, Followill DS. Pencil beam algorithms are unsuitable for proton dose calculations in lung. Int J Radiat Oncol Biol Phys 2017:99:750-6.

36. Huang $S$, Souris K, Li S, et al. Validation and application of a fast Monte Carlo algorithm for assessing the clinical impact of approximations in analytical dose calculations for pencil beam scanning proton therapy. Med Phys 2018;45:5631-42.

37. De Oliveira Duarte $S$, Rancoule C, He MY, et al. Use of 4D-CT for radiotherapy planning and reality in France: data from a national survey. Cancer Radiother 2019;23:395-400.

38. Pan T, Lee TY, Rietzel E, Chen GT. 4D-CT imaging of a volume influenced by respiratory motion on multi-slice CT. Med Phys 2004;31:333-40.

39. Martin R, Pan T. Target volume and artifact evaluation of a new data-driven 4D CT. Pract Radiat Oncol 2017;7:e345-54.

40. Keall PJ, Mageras GS, Balter JM, et al. The management of respiratory motion in radiation oncology report of AAPM Task Group 76. Med Phys 2006;33:3874-900.

41. Zhang Y, Huth I, Weber DC, Lomax AJ. Dosimetric uncertainties as a result of temporal resolution in 4D dose calculations for PBS proton therapy. Phys Med Biol 2019;64:125005.

42. George R, Chung TD, Vedam SS, et al. Audio-visual biofeedback for respiratory-gated radiotherapy: impact of audio instruction and audio-visual biofeedback on respiratory-gated radiotherapy. Int J Radiat Oncol Biol Phys 2006;65:924-33.

43. Li R, Lewis JH, Cervino LI, Jiang SB. 4D CT sorting based on patient internal anatomy. Phys Med Biol 2009;54:4821-33.

44. Mori S, Hirai R, Sakata Y. Using a deep neural network for four-dimensional CT artifact reduction in image-guided radiotherapy. Phys Med 2019;65:67-75.

45. Matney J, Park PC, Bluett J, et al. Effects of respiratory motion on passively scattered proton therapy versus intensity modulated photon therapy for stage III lung cancer: are proton plans more sensitive to breathing motion? Int J Radiat Oncol Biol Phys 2013;87:576-82.

46. Bert C, Grozinger SO, Rietzel E. Quantification of interplay effects of scanned particle beams and moving targets. Phys Med Biol 2008;53:2253-65.

47. Boria AJ, Uh J, Pirlepesov F, et al. Interplay effect of target motion and pencil-beam scanning in proton therapy for pediatric patients. Int J Part Ther 2018;5:1-10.

48. Grassberger C, Dowdell S, Lomax A, et al. Motion interplay as a function of patient parameters and spot size in spot scanning proton therapy for lung cancer. Int J Radiat Oncol Biol Phys 2013;86:380-6.

49. Dowdell S, Grassberger C, Sharp GC, Paganetti H. Interplay effects in proton scanning for lung: a 4D Monte Carlo study assessing the impact of tumor and beam delivery parameters. Phys Med Biol 2013;58:4137-56.

50. Seco J, Robertson D, Trofimov A, Paganetti H. Breathing interplay effects during proton beam scanning: simulation and statistical analysis. Phys Med Biol 2009;54:N283-94.

51. Xing $Y$, Wu $X$, Li Y, Zhao J. Homogeneity study of proton and carbon ion scanning beams using combinations of different spot sizes and grid sizes. Med Phys 2017;44:6047-52.

52. Li Y, Kardar L, Li $X$, et al. On the interplay effects with proton scanning beams in stage III lung cancer. Med Phys 2014;41:021721.

53. Grassberger C, Dowdell S, Sharp G, Paganetti H. Motion mitigation for lung cancer patients treated with active scanning proton therapy. Med Phys 2015;42:2462-9.

54. Park PC, Zhu XR, Lee AK, et al. A beam-specific planning target volume (PTV) design for proton therapy to account for setup and range uncertainties. Int J Radiat Oncol Biol Phys 2012;82:e329-36.

55. Rietzel $E$, Bert $C$. Respiratory motion management in particle therapy. Med Phys 2010;37:449-60.

56. Lin L, Kang M, Huang $S$, et al. Beam-specific planning target volumes incorporating 4D CT for pencil beam scanning proton therapy of thoracic tumors. J Appl Clin Med Phys 2015;16:5678.

57. Graeff $C$, Durante $M$, Bert C. Motion mitigation in intensity modulated particle therapy by internal target volumes covering range changes. Med Phys 2012;39:6004-13.

58. Unkelbach J, Chan TC, Bortfeld T. Accounting for range uncertainties in the optimization of intensity modulated proton therapy. Phys Med Biol 2007;52:2755-73.

59. Fredriksson A, Forsgren A, Hardemark B. Minimax optimization for handling range and setup uncertainties in proton therapy. Med Phys 2011;38:1672-84.

60. Pflugfelder D, Wilkens JJ, Oelfke U. Worst case optimization: a method to account for uncertainties in the optimization of intensity modulated proton therapy. Phys Med Biol 2008;53:1689-700.

61. Liu W, Li Y, Li X, Cao W, Zhang X. Influence of robust optimization in intensity-modulated proton therapy with different dose delivery techniques. Med Phys 2012;39:3089101.

62. Fredriksson A, Bokrantz R. A critical evaluation of worst case optimization methods for robust intensity-modulated proton 
therapy planning. Med Phys 2014;41:081701.

63. Chen W, Unkelbach J, Trofimov A, et al. Including robustness in multi-criteria optimization for intensity-modulated proton therapy. Phys Med Biol 2012;57:591-608.

64. Graeff C. Motion mitigation in scanned ion beam therapy through 4D-optimization. Phys Med 2014;30:570-7.

65. Ge $S$, Wang $X$, Liao $Z$, et al. Potential for improvements in robustness and optimality of intensity-modulated proton therapy for lung cancer with 4-dimensional robust optimization. Cancers (Basel) 2019;11: E35.

66. Liu W, Schild SE, Chang JY, et al. Exploratory study of 4D versus $3 \mathrm{D}$ robust optimization in intensity modulated proton therapy for lung cancer. Int J Radiat Oncol Biol Phys 2016;95:523-33.

67. Graeff C, Luchtenborg R, Eley JG, Durante M, Bert C. A 4D-optimization concept for scanned ion beam therapy. Radiother Oncol 2013;109:419-24.

68. Furukawa $T$, Inaniwa $T$, Sato $S$, et al. Moving target irradiation with fast rescanning and gating in particle therapy. Med Phys 2010;37:4874-9.

69. Engwall E, Fredriksson A, Glimelius L. 4D robust optimization including uncertainties in time structures can reduce the interplay effect in proton pencil beam scanning radiation therapy. Med Phys 2018 Jul 16 [Epub]. https://doi.org/10.1002/ mp.13094.

70. Huang TC, Wang YC, Chiou YR, Kao CH. Respiratory motion reduction in $\mathrm{PET} / \mathrm{CT}$ using abdominal compression for lung cancer patients. PLoS One 2014;9:e98033.

71. Rasheed A, Jabbour SK, Rosenberg S, et al. Motion and volumetric change as demonstrated by 4DCT: the effects of abdominal compression on the GTV, lungs, and heart in lung cancer patients. Pract Radiat Oncol 2016;6:352-9.

72. Bouilhol G, Ayadi M, Rit $S$, et al. Is abdominal compression useful in lung stereotactic body radiation therapy?: a 4DCT and dosimetric lobe-dependent study. Phys Med 2013;29:33340.

73. Mampuya WA, Nakamura M, Matsuo $Y$, et al. Interfraction variation in lung tumor position with abdominal compression during stereotactic body radiotherapy. Med Phys 2013;40:091718.

74. Han C, Sampath S, Schultheisss TE, Wong JY. Variations of target volume definition and daily target volume localization in stereotactic body radiotherapy for early-stage non-small cell lung cancer patients under abdominal compression. Med Dosim 2017;42:116-21.

75. Hanley J, Debois MM, Mah D, et al. Deep inspiration breathhold technique for lung tumors: the potential value of target immobilization and reduced lung density in dose escalation. Int J Radiat Oncol Biol Phys 1999;45:603-11.

76. Scherman Rydhog J, Riisgaard de Blanck S, Josipovic M, et al. Target position uncertainty during visually guided deepinspiration breath-hold radiotherapy in locally advanced lung cancer. Radiother Oncol 2017;123:78-84.

77. Josipovic M, Persson GF, Dueck J, et al. Geometric uncertainties in voluntary deep inspiration breath hold radiotherapy for locally advanced lung cancer. Radiother Oncol 2016;118:5104.

78. Josipovic M, Aznar MC, Thomsen JB, et al. Deep inspiration breath hold in locally advanced lung cancer radiotherapy: validation of intrafractional geometric uncertainties in the INHALE trial. Br J Radiol 2019;92:20190569.

79. Lee $D$, Greer PB, Lapuz C, et al. Audiovisual biofeedback guided breath-hold improves lung tumor position reproducibility and volume consistency. Adv Radiat Oncol 2017;2:354-62.

80. Gorgisyan J, Munck Af Rosenschold P, et al. Feasibility of pencil beam scanned intensity modulated proton therapy in breath-hold for locally advanced non-small cell lung cancer. Int J Radiat Oncol Biol Phys 2017;99:1121-8.

81. Wong JW, Sharpe MB, Jaffray DA, et al. The use of active breathing control $(A B C)$ to reduce margin for breathing motion. Int J Radiat Oncol Biol Phys 1999;44:911-9.

82. Panakis N, McNair HA, Christian JA, et al. Defining the margins in the radical radiotherapy of non-small cell lung cancer (NSCLC) with active breathing control (ABC) and the effect on physical lung parameters. Radiother Oncol 2008;87:65-73.

83. Brock J, McNair HA, Panakis N, Symonds-Tayler R, Evans PM, Brada M. The use of the Active Breathing Coordinator throughout radical non-small-cell lung cancer (NSCLC) radiotherapy. Int J Radiat Oncol Biol Phys 2011;81:369-75.

84. Lu L, Diaconu C, Djemil T, et al. Intra- and inter-fractional liver and lung tumor motions treated with SBRT under active breathing control. J Appl Clin Med Phys 2018;19:39-45.

85. Kaza E, Dunlop A, Panek $R$, et al. Lung volume reproducibility under $A B C$ control and self-sustained breath-holding. J Appl Clin Med Phys 2017;18:154-62.

86. Den Otter LA, Kaza E, Kierkels RGJ, et al. Reproducibility of the lung anatomy under active breathing coordinator control: dosimetric consequences for scanned proton treatments. Med Phys 2018;45:5525-34.

87. Matsuura T, Miyamoto N, Shimizu S, et al. Integration of a real-time tumor monitoring system into gated proton spotscanning beam therapy: an initial phantom study using patient tumor trajectory data. Med Phys 2013;40:071729.

88. Kanehira T, Matsuura T, Takao S, et al. Impact of real-time 
image gating on spot scanning proton therapy for lung tumors: a simulation study. Int J Radiat Oncol Biol Phys 2017;97:173-81.

89. Ciocca M, Mirandola $A$, Molinelli $S$, et al. Commissioning of the 4-D treatment delivery system for organ motion management in synchrotron-based scanning ion beams. Phys Med 2016;32:1667-71.

90. Yamada T, Miyamoto N, Matsuura T, et al. Optimization and evaluation of multiple gating beam delivery in a synchrotronbased proton beam scanning system using a real-time imaging technique. Phys Med 2016;32:932-7.

91. Schatti A, Zakova M, Meer D, Lomax AJ. Experimental verification of motion mitigation of discrete proton spot scanning by re-scanning. Phys Med Biol 2013;58:8555-72.

92. Bernatowicz K, Lomax AJ, Knopf A. Comparative study of layered and volumetric rescanning for different scanning speeds of proton beam in liver patients. Phys Med Biol 2013;58:7905-20.

93. Schatti A, Meer D, Lomax AJ. First experimental results of motion mitigation by continuous line scanning of protons. Phys Med Biol 2014;59:5707-23.

94. Riboldi M, Orecchia R, Baroni G. Real-time tumour tracking in particle therapy: technological developments and future perspectives. Lancet Oncol 2012;13:e383-91.

95. Particle Therapy Co-Operative Group. Statistics of patients treated in particle therapy facilities worldwide [Internet] Villigen, Switzerland: Particle Therapy Co-Operative Group; c2019 [cited 2019 Dec 26]; Available from: https://www.ptcog. ch/index.php/ptcog-patient-statistics.

96. Particle Therapy Co-Operative Group. Particle therapy facilities in clinical operation [Internet]. Villigen, Switzerland: Particle Therapy Co-Operative Group; c2019 [cited 2019 Dec 26]. Available from: https://www.ptcog.ch/index.php/facilities-inoperation.

97. Oh D. Proton therapy: the current status of the clinical evidences. Precis Future Med 2019;3:91-102. 